Relations industrielles

Industrial Relations

\title{
Studies in Personnel and Industrial Psychology (Revised Edition), by Edwin A. Fleishman, Dorsey, 1967, 801 pages.
}

\section{Jean Boivin}

Volume 22, numéro 4, 1967

URI : https://id.erudit.org/iderudit/027856ar

DOI : https://doi.org/10.7202/027856ar

Aller au sommaire du numéro

Éditeur(s)

Département des relations industrielles de l'Université Laval

ISSN

0034-379X (imprimé)

1703-8138 (numérique)

Découvrir la revue

Citer ce compte rendu

Boivin, J. (1967). Compte rendu de [Studies in Personnel and Industrial Psychology (Revised Edition), by Edwin A. Fleishman, Dorsey, 1967, 801 pages.] Relations industrielles / Industrial Relations, 22(4), 590-591.

https://doi.org/10.7202/027856ar

Tous droits réservés @ C Département des relations industrielles de l'Université Laval, 1967
Ce document est protégé par la loi sur le droit d'auteur. L’utilisation des services d'Érudit (y compris la reproduction) est assujettie à sa politique d'utilisation que vous pouvez consulter en ligne.

https://apropos.erudit.org/fr/usagers/politique-dutilisation/ 
The changes in functions in collective bargaining and the necessity to evaluate the admitted benefits of this industrial institution and the potential dangers arising from its limitations are critically examined in the light of past and present economic conditions.

The matters which comprise the subject of the book are placed in an american setting and center about the industrial scene in the United States.

Nevertheless, there is a great deal of analysis of the different topics which will be of interest to students and practioners in industrial relations, everywhere, who are interested in principles and developments in that area.

The reviewer commends this volume as a fruitful contribution to the literature of the sectors covered.

\section{Benjamin S. KIRSH}

A Manual for Employees-Management Co-operation in the Federal Service, by Horold S. Roberts, Industrial Relations Center, University of Hawaii, Honolulu, Hawaii, February 1967, 275 pages. (Third Edition.)

Le développement continuel des relations industrielles dans le fonctionnarisme fédéral oméricain n'a pas cessé depuis quelques onnées d'amener des éléments nouveaux. Depuis que le Président Kennedy a mandaté, en juin 1961, une équipe spéciale de recherches (Task Force) sur la question, divers documents officiels ont été émis. Le point de départ a été la remise du rapport de cette commission d'enquête.

Ce manuel « sur la coopération employésgérance dans le service fédéral » constitue en quelque sorte un recueil d'à-peu-près tous les documents officiels émis à date. Ces documents sont évidemment situés dans leur contexte et précédés d'explications, lorsque nécessaire.

Le choix de ces documents et leur disposition de façon claire et ordonnée donne a ce volume une valeur indiscutable pour quiconque est intéressé au développement d'un système de relations industrielles dans un service gouvernemental

L'intérêt plus large encore suscité par les développements récents ont incité les éditeurs à inclure dans leur publication certains textes ou commentaires spécifiques notom- ment les recommandations d'un panel sur les relations dans le fonctionnarisme au niveau d'un état (américain) et aussi les commentaires de personnalités telles le Secrétaire au Ministère du Travail, le Président de la Commission de la Fonction Publique et le Président de l'AFL-CIO

Après cette description sommaire du contenu nous laissons au lecteur le soin de décider lui-même jusqu'à quel point ce volume peut lui être utile.

\section{André PETIT}

Studies in Personnel and Industrial Psychology (Revised Edition), by Edwin A Fleishman, Dorsey, 1967, 801 pages.

L'auteur essaye d'inclure dans ce volume à la fois les avantoges d'un livre de base et l'intérêt d'un livre de lecture: générale II croit y arriver par une approche sélective, c'est-ò-dire en intégrant à chacune des sections des articles de revues écrits par des auteurs reconnus ( 81 au total), afin de mettre en évidence les études empiriques de base sur les différents aspects de la psychologie industrielle. Dans chacune des sections, l'auteur procède de la même manière: il écrit lui-même une introduction dans laquelle il montre l'apport pertinent de chaque article aux principaux problèmes du sujet général de la section, puis, il fait suivre les différents articles en question.

Quant au contenu du volume il essaye de couvrir autant les aspects traditionnels du sujet, tels: lo sélection, la formation et l'évaluation du personnel, que les aspects socioux et motivationnels, tels: les problèmes de leadership, de communications et de sotisfaction au travail. De plus, dans la mesure où elles pouvaient éclairer le domaine de la psychologie industrielle, des recherches dans des disciplines connexes, telles la science militaire et l'éducotion ont été également incluses dans cet ouvrage.

De façon plus particulière, les 10 sections couvrent les sujets suivants: 1) la sélection du personnel; 2) l'évaluation du rendement; 3) la formation des employés et des cadres de direction; 4) la motivation, les attitudes et la satisfaction au travail; 5) le leadership et le contrôle; 6) les communications et les comportements au sein de l'entreprise ; 7) la fatigue, la monotonie et les conditions de travail; 8) les accidents et la sécurité industrielle; 9) la psychologie oppliquée oux systèmes hommes-machines (engineering psychology; 10) la psychologie appliquée oux consommoteurs (consumer psychology) 
Le grand avantage de ce volume est de présenter un éventail des nombreuses sources de la psychologie industrielle ainsi que les contributions des principoux chercheurs dans ce domaine. II s'agit d'un volume très intéressont pour celui qui veut explorer de façon générale la psychologie industrielle et très utile sur le plan académique pour quiconque veut confronter les diverses théories en cours

\section{Jean BOIVIN}

\section{Rapport de la Commission Royale sur les Relations Employeurs-Employés dans les Services Publics du Nouveau-Brunswick, por Dr Soul J. Frankel, 1967, 111 pages}

Ce ropport nous présente l'étude menée par la Commission sur trois champs et en trois mouvements, tels qu'arrêtés dans la décret en conseil 66-496, du 29 juin 1966.

1) Etude d'abord des relations employeursemployés dans le Service Civil;

2) L'enseignement;

3) Les hôpitaux.

Le rapport étudie brièvement les tendances actuelles dans chacun des secteurs, les mémoires qui furent présentés d̀ la commission et les recommandations de cette dernière

En fait, la distinction des trois secteurs (Service Civil, Commissions Scolaires, Hôpitoux) s'otténuent énormément sous l'aspect des relations employeurs-employés quand le ropport signale la prise de responsabilité presque totale du financement de l'enseionement et des services hospitaliers. II s'agit donc de trois groupes d'employés du gouvernement.

Lo Commission d'enquête tient compte de cette situation, et propose sensiblement les mêmes législations et politiques oux relations entre employeurs et employés touchant lo syndicalisation (unités de négociation), la négociation (procédure), la convention collective. Elle tient compte cependant de l'évolution et de l'organisation de ces trois secteurs selon la législation et les circonstances particulières de l'organisation professionnelle de ces groupes.

Le rapport décrit les principaux problemes de la syndicalisation du secteur public et désigne les forces en présence. Le problème est posé quant à l'ancienne législation (Loi des Relations du Travail), aux organismes para-syndicaux et associations professionnelles déjò existantes, oux diffé- rents employeurs: Etat, Corporations et Commissions de la Couronne, Association Hospitalière et Association des Commissaires d'Ecoles

Enfin, tout un processus de négociotion ollant de la discussion permanente d lo grève est défini, de même que la distinction des unités de négociation, selon les caractères communs et distincts des différentes cotégories professionnelles.

En annexe sont ajoutées d'excellentes statistiques qui décrivent l'élargissement des chomps d'action de l'Etat et la croissance des effectifs de la main-d'oeurre employés dans le secteur public

\section{L.-René PARENTEAU}

\section{The French Labor Courts: Judgment by}

Peers, par William H. MacPherson et Frederic Meyers, Institute of Lobor and Industrial Relations, University of Illinois, Urbana, 1966, 104 pages

Ayant constaté à plusieurs reprises que les méthodes utilisées en Europe pour régler les conflits de travail semblaient fort méconnues aux Etats-Unis, MacPherson et Meyers ont décidé d'effectuer une étude empirique sur le fonctionnement des conseils de prud'hommes, en France. Et, par souci d'objectivité, ils se sont limités à une analyse de cette institution plutôt qu'à la recherche d'une hypothétique supériorité d'un des systèmes sur l'autre.

Dans un premier chapitre consacré à l'introduction, les auteurs ont établi les objectifs qu'ils s'étaient fixés en entreprenont cette étude. Ils ont de plus précisé d̀ leurs lecteurs américains deux différences fondamentales entre le système de leur pays et celui qu'ils ont étudié:

1) le nombre d'avantoges sociaux dont bénéficie le travailleur français en vertu de la loi, alors que l'ouvrier américain doit encore, actuellement, en négocier en très grand nombre.

2) La présence exclusive, comme juges, de non juristes élus par les employeurs et les employés.

Après un deuxième chapitre relatant I'histoire des conseils de prud'hommes - vieille de plus de cent cinquante ans - les auteurs étudient, dans le troisième, lo structure, la compétence et la procédure actuelles de ces tribunaux. On doit noter qu'il existe actuellement environ deux cent cinquante 